\title{
Progranulin (PGRN) is serves as an inflammation-response biomarker and promotes lung damage in burn-induced Sepsis via the SIRT1 Pathways by ROS
}

\author{
Hui DING ${ }^{1}$, Zhaoling SHI ${ }^{1}$, Lu ZHANG ${ }^{1}$, Nairong GAO ${ }^{1 *}$, Xiaoning CHENG ${ }^{1}$, Haibo LIN $^{1}$, Zhihong ZHANG \\ Guocheng $\mathrm{ZHANG}^{1 *}$ (1)
}

\begin{abstract}
Sepsis has become the focus of research in the field of intensive care medicine due to its high mortality and complicated pathogenesis. To evaluate the effects and mechanism of Progranulin (PGRN) affects inflammation in lung damage of burninduced Sepsis. In mice of burn-induced Sepsis, PGRN gene expression was increased in lung tissue. So, PGRN promoted inflammation in vitro model through SIRT1/ROS/NLRP3 pathways. Down-regulation of PGRN reduced inflammation via SIRT1/ROS/NLRP3 Pathways. The activation of SIRT1 reduced the effects of PGRN on inflammation in lung cell by LPS via SIRT1/ROS/NLRP3 pathways. The inactivation of NLRP3 decreased the effects of PGRN on inflammation in vitro model. Our data suggest that PGRN is serves as inflammation-response biomarker and promoted lung damage in burninduced Sepsis via the SIRT1 Pathways by ROS/NLRP3 pathways.
\end{abstract}

Keywords: PGRN; sepsis; lung damage; SIRT1; ROS; NLRP3.

Practical Application: This study is of great importance for investigating the role of PGRN, serving as inflammation-response biomarker, in lung damage in burn-induced Sepsis.

\section{Introduction}

Sepsis is the systemic inflammatory response syndrome (SIRS) caused by bacterial infection (Jajoo et al., 2018). Sepsis is one of the common severe complications following burn trauma, shock, and operation in critically ill patients, which is also an important factor inducing multiple organ dysfunction syndrome (MODS) (Jajoo et al., 2018). Acute lung injury (ALI) is an acute hypoxic respiratory insufficiency caused by diffuse interstitial and alveolar edema due to various causes (Chen et al., 2018). Among the diverse causes of ALI, sepsis is considered the most important high-risk factor for ALI progression. The incidence of sepsis in ALI is high, with the mortality as high as $30-50 \%$ (Chen et al., 2018). Scholars have found that the average global mortality rate is $43 \%$ from 72 clinical studies, which poses a major threat to human health. In mainland China, approximately 59\% patients admitted to the ICU are due to infection, of whom, 37.3\% would progress into severe sepsis and septic shock, and 27.1\% would progress into ALI and ARDS (Dominguez et al., 2013).

Silent information regulator 1 (SIRT1) is a $\mathrm{NAD}^{+}$-dependent transcriptional regulator with histone deacetylase activity, exerting a variety of biological activities such as anti-inflammation and anti-apoptosis (Chen et al., 2016). Therefore, SIRT1 has gradually been the focus of research. Accumulative studies have shown that Sirtuins may be involved in changes of inflammation, immunity and metabolism during the sepsis, which is also closely associated with cell proliferation, differentiation, aging, apoptosis and metabolism (Chen et al., 2016; Huang et al., 2017).
Progranulin (PGRN) is a multifunctional secretory glycoprotein (Kim \& Choi, 2012). The abnormal changes of its expression and related genes are closely associated with inflammatory lesions of the nervous system, degenerative diseases, degenerative diseases, lysosomal homeostasis in the brain, and integrity of the bloodbrain barrier, which are involved in the pathological process of multiple nervous system diseases (Kim \& Choi, 2012). PGRN is a multifunctional protein that can promote cell proliferation, tumorigenesis, and regulate inflammatory responses (Jia et al., 2009). PGRN is widely involved in various diseases of the nervous system and other systems and plays an important role in the development of the nervous system (Zhu et al., 2014). To evaluate the effects and mechanism of PGRN affects inflammation in lung damage of burn-induced Sepsis.

\section{Materials and methods}

\subsection{Animal model}

This study was approved by the Institutional Animal Care and Use Committee of XX Hospital. Male mice at 5-6 week of age were anesthetized with $50 \mathrm{mg} / \mathrm{kg}$ pentobarbital sodium. An adjustable burn template that accounted for variation in body surface area was filled with boiling water at $40 \%$ total body surface full-thickness burn and placed on the dorsal and ventral skin for $10 \mathrm{~s} .1 \mu \mathrm{g}$ of PGRN recombinant protein was intraperitoneally injected into burn-induced mice. 
ELISA kits. Tissue sample was collected at $2000 \mathrm{~g}$ for $15 \mathrm{~min}$ at $4{ }^{\circ} \mathrm{C}$. ELISA kits was used to measure Serum TNF- $\alpha$, IL-18, IL- 6 and IL- $1 \beta$ levels. Cell was collected, lysed using RIPA lysis buffer with $1 \%$ protease inhibitor and was used to measure the TNF- $\alpha$, IL-18, IL- 6 and IL- $1 \beta$ levels using ELISA kits. ROS levels were measured using ROS kits (S0033, Beyotime).

\subsection{HE staining}

Whole left lung tissue samples were paraffin-embedded for $24 \mathrm{~h}$ and cut into $5-\mu \mathrm{m}$ thick tissue sections. Tissue sections were stained with HE sassy and viewed and photographed using an OLYMPUS BX60 microscope (Olympus Corp., Tokyo, Japan).

\subsection{Bronchoalveolar lavage (BALF) fluid}

The left lung was lavaged using $2.0 \mathrm{~mL}$ of ice-cold phosphate buffered saline (PBS) for 3 times and BAL fluid was immediately centrifuged at $2000 \mathrm{~g}$ for $15 \mathrm{~min}$ at $4{ }^{\circ} \mathrm{C}$. Supernatants were stored at $-80^{\circ} \mathrm{C}$ for cytokine and protein analysis and total protein content in BALF was determined by the Bradford method (Beyotime).

\subsection{Lung wet/dry weight ratio}

The right lung was excised and weight was determined for wet weight. The lung tissue was placed at $80{ }^{\circ} \mathrm{C}$ for $24 \mathrm{~h}$ and weight was determined for dry weight. The wet/dry weight ratios were then calculated.

\subsection{RNA extraction and real-time PCR and microarray}

Total RNA of samples was extracted from cells using Trizol ${ }^{\oplus}$ reagent (Ambion ${ }^{\circledR}$, LifeTechnologies). 50 ng of total RNA was performed to synthesis cDNA using the PrimeScript ${ }^{\circledR}$ RT reagent Kit with gDNA Eraser according to the manufacturer's instructions (TaKaRa Bio). Real-time PCR was performed using the ABI7500 System employing SYBR ${ }^{\circledR}$ Select Master Mix (Applied Biosystems ${ }^{\circledast}$, Life-Technologies). For microarray experiment, total RNA was extracted using the mirVana ${ }^{\mathrm{TM}}$ miRNA Isolation Kit (Ambion, Austin, TX, USA) and data was evaluated using an Agilent Bioanalyzer 2100 (Agilent Technologies, Santa Clara, CA, USA). Microarray analysis was performed by Shanghai Biotechnology Cooperation (Shanghai, P.R. China).

\subsection{Cell culture and plasmid construction}

Human non-small cell lung cancer A549 cell was grown in RPMI-1640 medium (Gibco, Thermo Fisher Scientific) contained 10\% fetal bovine serum (FBS) (Gibco, Thermo Fisher Scientific) at $37^{\circ} \mathrm{C}$ with 5\% CO2 95\% air. PGRN, siPGRN, SIRT1, siSIRT1 and negative mimics were transfected into cell using Lipofectamine ${ }^{\circledast} 2000$ Reagent (Invitrogen, Carlsbad, CA, USA) according to the manufacturer's instructions. After transfection for $4 \mathrm{~h}$, cell transfection was treated with $500 \mathrm{ng}$ of LPS and 2 $\mathrm{nM}$ of ATP for lung damage model.

\subsection{Western blot analysis}

Briefly, whole cells collected and lysed using RIPA lysis buffer with $1 \%$ protease inhibitor. Then, The bicinchoninic acid reagent (BCA) was used quantified protein concentration. Protein samples of every group were separated by $10 \%$ SDSPAGE and transferred onto PVDF membranes. Membranes were incubated with primary antibody against PGRN, SIRT1, NLRP3 and GAPDH (Cell Signaling Technology, USA) overnight at $4{ }^{\circ} \mathrm{C}$ after blocked in 5\% non-fat milk for $2 \mathrm{~h}$. After membrane was washed with PBS, membranes were incubated with horseradish peroxidase-conjugated secondary antibodies at room temperature for $1 \mathrm{~h}$. The protein bands were detected using enhanced chemiluminescence (ECL) reagent, and quantified using Image Lab Software 3.0 (Bio-Rad Laboratories, Berkeley, CA, USA).

Statistical analysis. Differences of these date were considered statistically significant in this experiment if $\mathrm{P}<0.05$. All results of this experiment are expressed as mean \pm standard deviation (S.D.). Student's t-test or one-way analysis of variance (ANOVA) and Tukey's post test was used for comparisons between groups.

\section{Results}

\subsection{PGRN promoted inflammation and accelerated lung injury of burn-induced sepsis}

To investigate the effects and mechanism of lung damage of burn-induced Sepsis, this experiment analyzed the expression of PGRN gene in lung tissue. As $12 \mathrm{~h}$ and $24 \mathrm{~h}$ of burn model, PGRN mRNA expression was up-regulated in burn model of mice (Figure 1A). Then, PGRN protein expression in burn model were induced (Figure 1B, 1C). Next, PGRN recombinant protein were injected into burn model, and survival rate were decreased in PGRN group, compared with burn model mice group (Figure 1D). Lung W/D rate and protein content of bronchoalveolar lavage were peaking, and HE staining that lung cell had large death in PGRN group, compared with burn model mice group (Figure $1 \mathrm{E}-\mathrm{G}$ ). Then, Inflammatory factors (TNF- $\alpha$, IL-1 $\beta$, IL-6 and IL-18 levels) of tissue samples were increased in PGRN group, comparing with burn model mice group (Figure $1 \mathrm{H}-\mathrm{K}$ ).

\subsection{PGRN regulated inflammation in vitro model}

To analyzed the effects of PGRN on inflammation in vitro model of Sepsis, over-expression of PGRN promoted TNF- $\alpha$, IL-1 $\beta$, IL-6 and IL-18 levels, and increased ROS production levels of LPS+ATP-induced vitro model (Figure 2A-F). Down-regulation of PGRN reduced LPS+ATP-induced cell TNF- $\alpha$, IL-1 $\beta$, IL- 6 and IL-18 levels, and ROS production levels vitro model (Figure $2 \mathrm{G}-\mathrm{L}$ ).

\subsection{PGRN targeted SIRT1 in burn-induced sepsis}

To explore the mechanism of PGRN on inflammation in vitro model. As shown in Figure 3A, SIRT1 maybe an target spot of PGRN on inflammation in burn-induced sepsis. PGRN overexpression induced PGRN protein expression, and suppressed SIRT1 protein expression in vitro mode of Sepsis (Figure 3B-D). Si-SIRT1 suppressed PGRN protein expression and induced SIRT1 protein expression in vitro mode of Sepsis (Figure 3E-G). 

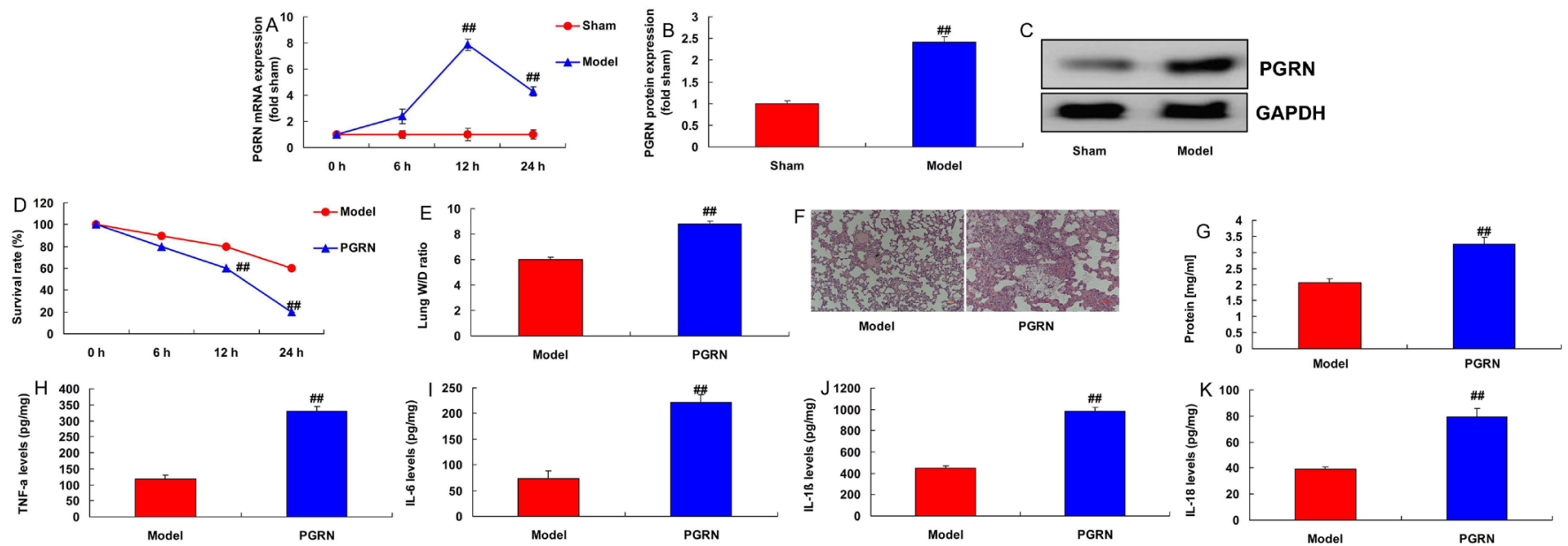

Figure 1. PGRN promoted inflammation and accelerated lung injury of burn-induced Sepsis. PGRN mRNA (A) and protein expression (B and C) in lung tissue of burn-induced Sepsis; survival rate (D), lung W/D rate (E), HE staining (F), protein content of bronchoalveolar lavage (G), TNF- $\alpha$ (H), IL-6 (I), IL-1ß (J) and IL-18 (K) levels in burn-induced Sepsis by PGRN. Sham, sham control group; Model, lung injury of burn-induced Sepsis group; PGRN, lung injury of burn-induced Sepsis by treatment with PGRN group. ${ }^{\# \#} \mathrm{p}<0.01$ compared with sham control or model group.
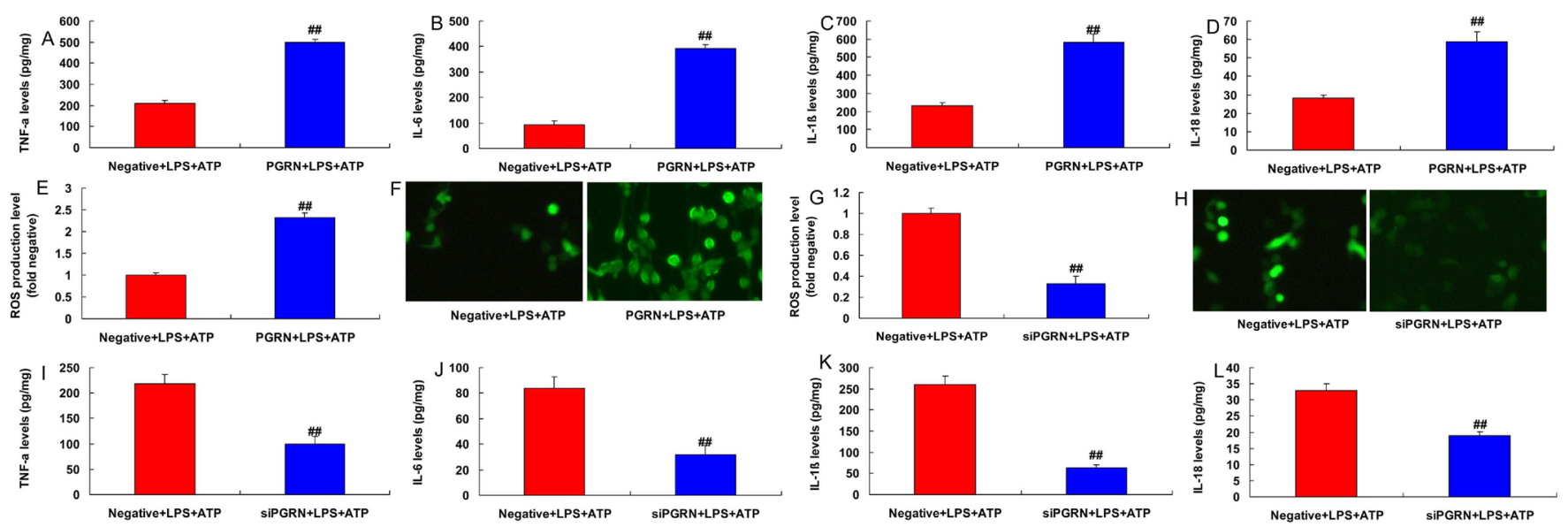

Figure 2. PGRN regulated inflammation in vitro model. TNF- $\alpha$ (A), IL-6 (B), IL-1 $\beta$ (C) and IL-18 (D) levels, ROS production levels (E and F) in vitro model by over-expression of PGRN; ROS production levels (G and H), TNF- $\alpha$ (I), IL-6 (J), IL-1 $\beta$ (K) and IL-18 (L) levels in vitro model by down-regulation of PGRN. Negative, negative control group; PGRN, over-expression of PGRN group; siPGRN, down-regulation of PGRN group. ${ }^{\# \#} \mathrm{p}<0.01$ compared with negative control group.

\subsection{SIRT1 reduced the effects of PGRN in lung tissue of burn- induced sepsis in vitro model}

To further confirm the role of SIRT1 in the effects of PGRN on Sepsis-induced inflammation in vitro model. Si-sirt1 suppressed SIRT1 protein expression, reduced ROS production levels and inhibited TNF- $\alpha$, IL-1 $\beta$, IL- 6 and IL-18 levels in vitro model of sepsis (Figure 4A-H). Over-expression of sirt1 induced SIRT1 protein expression, promoted ROS production levels and increased TNF- $\alpha$, IL-1 $\beta$, IL- 6 and IL- 18 levels in vitro model of sepsis (Figure 4I-P).

\subsection{SIRT1 reduced the effects of PGRN in lung tissue of burn- induced sepsis by NLRP3 inflammasome}

To further explore the mechanism of SIRT1 in the effects of PGRN on inflammation in vitro model. This experiment used gene chip to screen out the target spot of SIRT1 in the effects of PGRN on inflammation in burn-induced Sepsis. As shown in Figure 5A, NLRP3 maybe an target spot of SIRT1 in the effects of PGRN on inflammation in burn-induced sepsis. PGRN over-expression induced NLRP3 protein expression in vitro mode of Sepsis and si-PGRN suppressed NLRP3 protein expression in vitro mode of Sepsis (Figure 5B-E). SIRT1 over-expression suppressed NLRP3 protein expression in vitro mode of Sepsis and si-PGRN induced NLRP3 protein expression in vitro mode of Sepsis (Figure 5F-H). Meanwhile, we used vivo model and found that SIRT1 activator (CAY10602) recovered survival rate, and reduced lung W/D rate and protein content of bronchoalveolar lavage, and protected the damaging lung cell in burn-induced Sepsis by PGRN, comparing with treatment with PGRN group (Figure 6A-D). SIRT1 activator induced SIRT1 protein expression, suppressed NLRP3 protein expression, and reduced TNF- $\alpha$, IL-18, IL-6 and IL-1 $\beta$ levels in burn-induced Sepsis by PGRN, comparing with treatment with PGRN group (Figure 6E-K). 


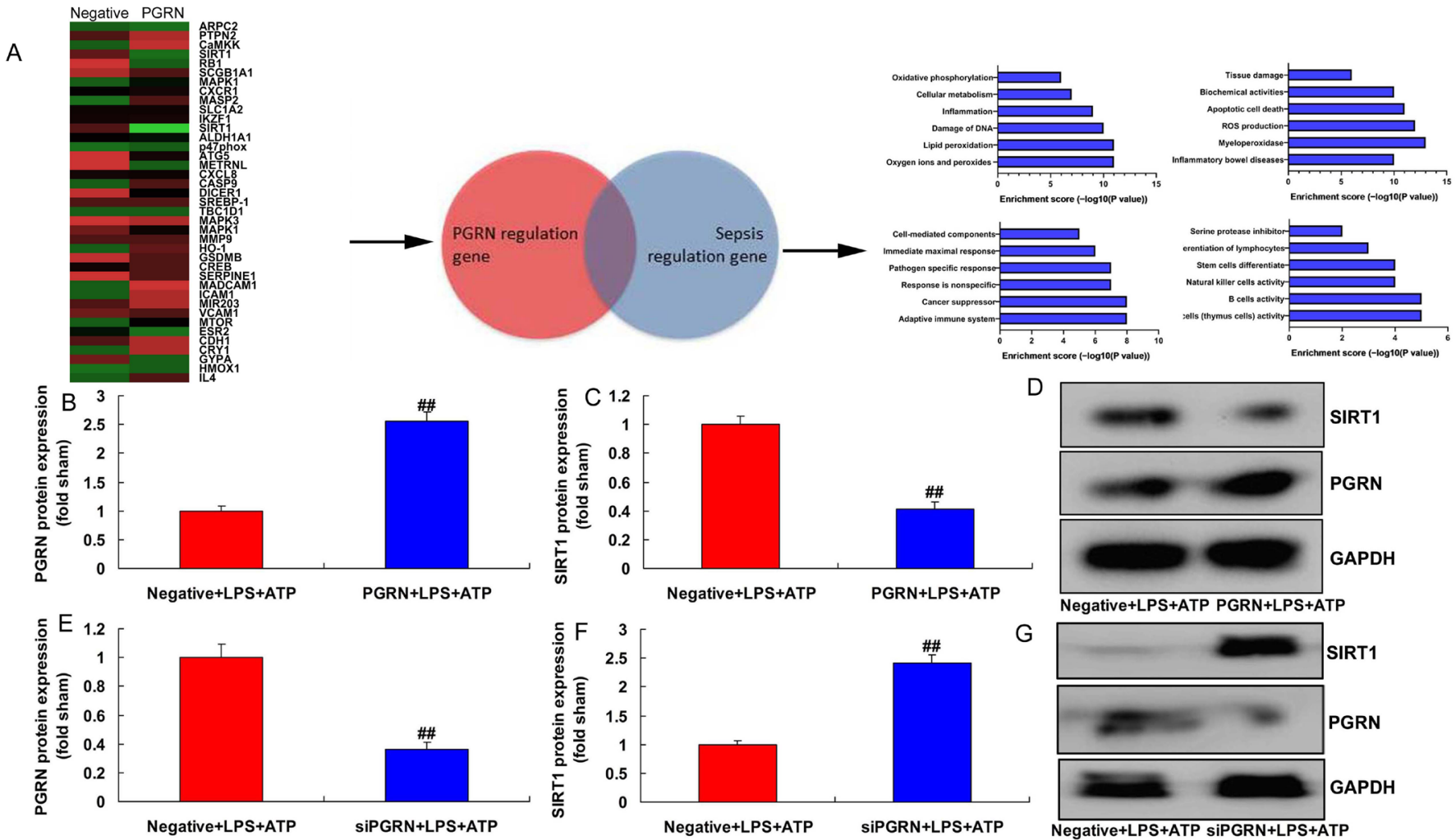

Figure 3. PGRN targeted SIRT1 in burn-induced Sepsis. Analysis results (A), PGRN and SIRT1 protein expressions (B, C and D) in vitro model by over-expression of PGRN; PGRN and SIRT1 protein expressions (E, F and G) in vitro model by down-regulation of PGRN. Negative, negative control group; PGRN, over-expression of PGRN group; siPGRN, down-regulation of PGRN group. ${ }^{* \#} \mathrm{p}<0.01$ compared with negative control group.
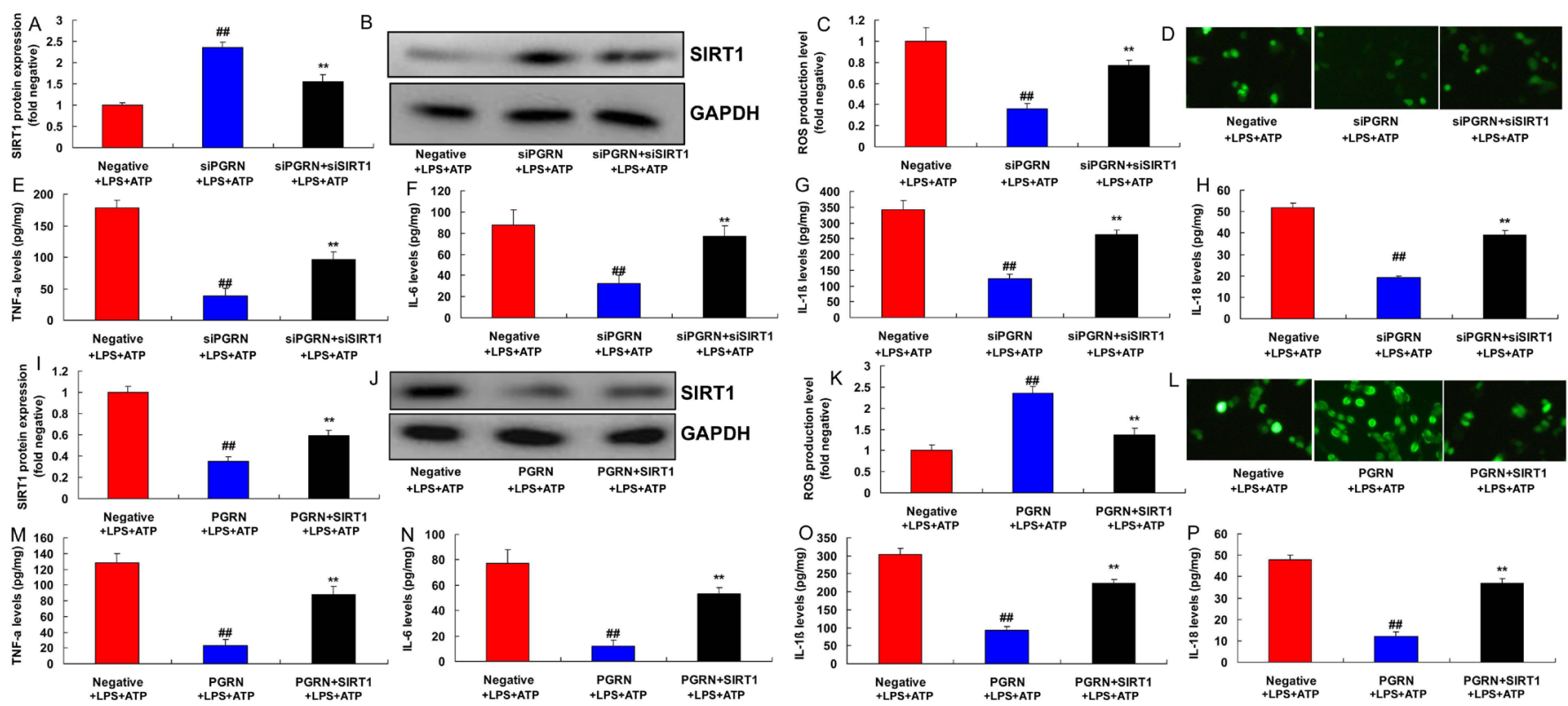

Figure 4. SIRT1 reduced the effects of PGRN in lung tissue of burn-induced Sepsis in vitro model. SIRT1 protein expressions (A and B), ROS production levels (C and D), TNF- $\alpha(E)$, IL-6 (F), IL-1 $\beta(G)$ and IL-18 (H) levels in vitro model by down-regulation of SIRT1; SIRT1 protein expressions (I and J), ROS production levels (K and L), TNF- $\alpha(\mathrm{M})$, IL-6 (N), IL-1 $\beta(\mathrm{O})$ and IL-18 (P) levels in vitro model by over-expression of SIRT1. Negative, negative control group; PGRN, over-expression of PGRN group; siPGRN, down-regulation of PGRN group; SIRT1, overexpression of SIRT1 group; si SIRT1, down-regulation of SIRT1 group. ${ }^{* *} \mathrm{p}<0.01$ compared with negative control group; ${ }^{* *} \mathrm{p}<0.01$ compared with PGRN or si PGRN group. 


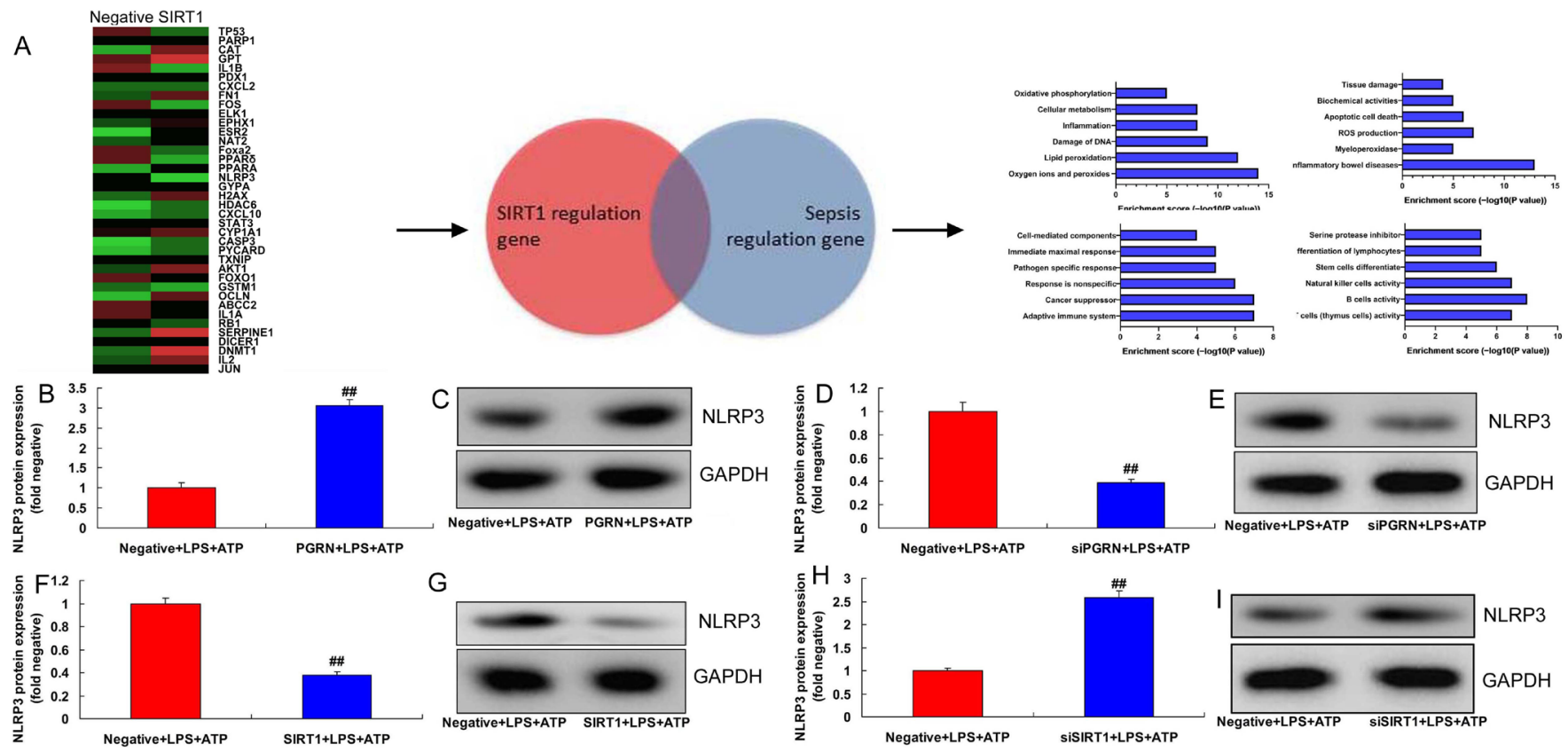

Figure 5. SIRT1 reduced the effects of PGRN in vitro model by NLRP3 inflammasome. Analysis results (A), NLRP3 protein expression in vitro model by over-expression of PGRN (B and C), NLRP3 protein expression in vitro model by down-regulation of PGRN (D and E), NLRP3 protein expression in vitro model by over-expression of SIRT1 (F and G), NLRP3 protein expression in vitro model by down-regulation of SIRT1 (H and I). Negative, negative control group; PGRN, over-expression of PGRN group; siPGRN, down-regulation of PGRN group; SIRT1, over-expression of SIRT1 group; si SIRT1, down-regulation of SIRT1 group. ${ }^{* \#} \mathrm{p}<0.01$ compared with negative control group.
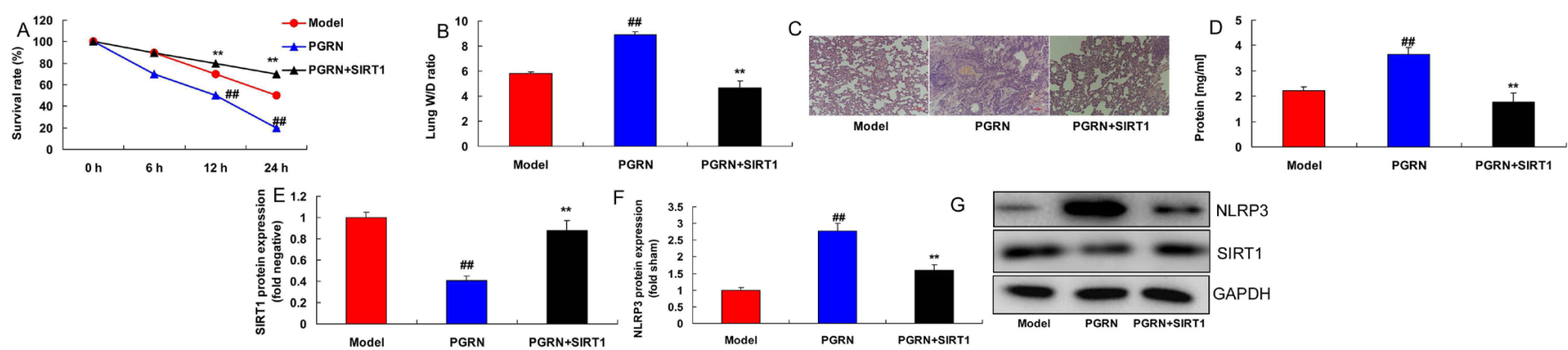

G
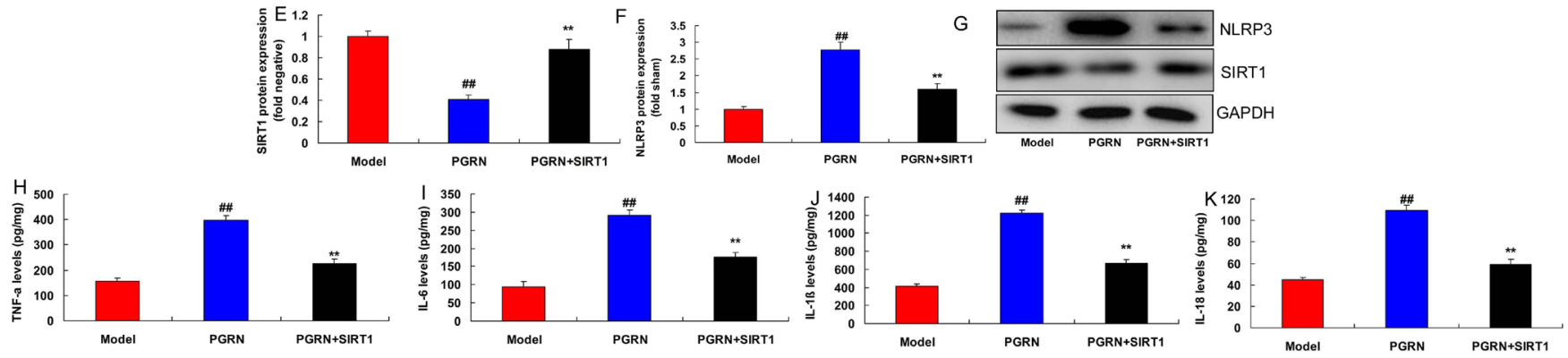

Figure 6. SIRT1 reduced the effects of PGRN in lung tissue of burn-induced Sepsis by NLRP3 inflammasome. Survival rate (A), lung W/D rate (B), HE staining (C), protein content of bronchoalveolar lavage (D), SIRT1/NLRP3 protein expression (E, F and G), TNF- $\alpha$ (H), IL-6 (I), IL-1 $\beta$ (J) and IL-18 (K) levels in burn-induced Sepsis by PGRN. Sham, sham control group; Model, lung injury of burn-induced Sepsis group; PGRN, lung injury of burn-induced Sepsis by treatment with PGRN group; SIRT1, SIRT1 activator (CAY10602) group. ${ }^{\#}$ p $<0.01$ compared with sham control or model group, ${ }^{* *} \mathrm{p}<0.01$ compared with PGRN group.

\section{Discussion}

Sepsis is the SIRS caused by various pathogenic factors (Newton et al., 2016). The high morbidity and high mortality of sepsis have made sepsis as a major problem that plagues clinical medical and scientific staffs worldwide (Newton et al., 2016). During the development of sepsis, multiple organs such as lungs, kidneys, and liver can be involved, which could further progress into MODS in the later stage (Marik, 2014). In addition, lung is the most vulnerable target organ after the occurrence of sepsis, generally with the earliest onset, which is manifested as
ALI/ARDS, with clinical manifestations of respiratory distress, refractory hypoxemia and respiratory failure (Marik, 2014). In the present study, PGRN promoted inflammation and accelerated lung injury of burn-induced Sepsis. Petry et al. (2014) reported that Progranulin is a novel biomarker in sepsis (Petry et al., 2014). Thus, H PGRN could observably impact the lung damage in burn-induced sepsis.

SIRT1 is a histone deacetylase of Sirtuin family widely existing in human cells, and is involved in the regulation of various physiological functions such as glucose and lipid 
metabolism and aging (Marik, 2014; Zhang et al., 2018). Recent studies have shown that SIRT1 can exert antiinflammatory effects by inhibiting multiple cell signaling pathways during the inflammatory response (Marik, 2014; Wei et al., 2019; Pedrazza et al., 2014). Our study demonstrated that PGRN targeted SIRT1 in burn-induced Sepsis by NLRP3. Livak \& Schmittgen (2001) showed that PGRN exerts inflammatory effects via SIRT1-NF- $\kappa B$ in adipose insulin resistance (Livak \& Schmittgen, 2001). PGRN might indicate the oncogenic role of NLRP3 in lung damage in burn-induced sepsis and provides creativity for in-inflammation exploration.

PGRN-mediated collagen-induced anti-arthritis effects are mainly achieved by the anti-inflammatory factor IL-10 (Kim \& Choi, 2012). IL-10 is an anti-inflammatory factor, and its expression level is correlated with the degree of inflammation (Jia et al., 2009; Wang et al., 2017). The serum level of IL-10 is higher in mice treated with PGRN for arthritis, while levels of IL-17, IL- 6 and TNF- $\alpha$ are greatly reduced (Jiang et al., 2015). Compared with wild-type macrophages, PGRN-deficient macrophages produce more pro-inflammatory cytokines and less anti-inflammatory factors IL-10 in response to lipopolysaccharide induction. In relevant studies on femoral head necrosis, PGRN can inhibit the inflammatory effects of TNF- $\alpha$ by binding to TNF receptors, weakening cartilage degradation and thereby exerting a protective role on bone (Jiang et al., 2015). This experiments revealed that SIRT1 reduced the effects of PGRN in lung tissue of burn-induced Sepsis by NLRP3 inflammasome. Ma et al. (2017) indicated that Progranulin opened inflammation and lung injury in mouse model of endotoxic shock (Ma et al., 2017). Therefore, PGRN is important in regulating gene expression through affecting inflammation by SIRT1/ROS/ NLRP3.

\section{Conclusion}

In summary, these results of this study revealed that PGRN is serves as an inflammation-response biomarker and promoted lung damage in burn-induced Sepsis via the SIRT1/ ROS/NLRP3 pathways. These findings suggest that PGRN gene function and molecular mechanism related to PGRN may provide a potential therapeutic candidate for lung damage in burn-induced Sepsis.

\section{Acknowledgements}

This study was supported by Subject Innovation Team of the Second Hospital Affiated Shaanxi University of Chinese Medicine. This research was supported by project of Subject Innovation Team of the Second Hospital Affifiliated Shaanxi University of Chinese Medicine (2020XKTD-A02).

\section{References}

Chen, G. D., Yu, W. D., \& Chen, X. P. (2016). SirT1 activator represses the transcription of TNFalpha in THP1 cells of a sepsis model via deacetylation of H4K16. Molecular Medicine Reports, 14(6), 5544-5550. http://dx.doi.org/10.3892/mmr.2016.5942. PMid:27878240.
Chen, P. H., Liu, A. J., Ho, K. H., Chiu, Y. T., Lin, Z. H. A., Lee, Y. T., Shih, C. M., \& Chen, K. C. (2018). microRNA-199a/b-5p enhance imatinib efficacy via repressing WNT2 signaling-mediated protective autophagy in imatinib-resistant chronic myeloid leukemia cells. Chemico-Biological Interactions, 291, 144-151. http://dx.doi. org/10.1016/j.cbi.2018.06.006. PMid:29890129.

Dominguez, J. A., Samocha, A. J., Liang, Z., Burd, E. M., Farris, A. B., \& Coopersmith, C. M. (2013). Inhibition of IKKbeta in enterocytes exacerbates sepsis-induced intestinal injury and worsens mortality. Critical Care Medicine, 41(10), e275-e285. http://dx.doi.org/10.1097/ CCM.0b013e31828a44ed. PMid:23939348.

Huang, J., Tian, R., Yang, Y., Jiang, R., Dai, J., Tang, L., \& Zhang, L. (2017). The SIRT1 inhibitor EX-527 suppresses mTOR activation and alleviates acute lung injury in mice with endotoxiemia. Innate Immunity, 23(8), 678-686. http://dx.doi.org/10.1177/1753425917733531. PMid:28952835.

Jajoo, M., Manchanda, V., Chaurasia, S., Sankar, M. J., Gautam, H., Agarwal, R., Yadav, C. P., Aggarwal, K. C., Chellani, H., Ramji, S., Deb, M., Gaind, R., Kumar, S., Arya, S., Sreenivas, V., Kapil, A., Mathur, P., Rasaily, R., Deorari, A. K., \& Paul, V. K. (2018). Alarming rates of antimicrobial resistance and fungal sepsis in outborn neonates in North India. PLoS One, 13(6), e0180705. http://dx.doi.org/10.1371/ journal.pone.0180705. PMid:29953451.

Jia, G., Mitra, A. K., Gangahar, D. M., \& Agrawal, D. K. (2009). Regulation of cell cycle entry by PTEN in smooth muscle cell proliferation of human coronary artery bypass conduits. Journal of Cellular and Molecular Medicine, 13(3), 547-554. http://dx.doi.org/10.1111/j.15824934.2008.00384.x. PMid:18544045.

Jiang, H., Wang, L., \& Pan, J. (2015). Inhibition of pro-protein convertase subtilisin/kexin type 6 has a protective role against synovitis in a rat model of rheumatoid arthritis. Molecular Medicine Reports, 12(5), 7681-7686. http://dx.doi.org/10.3892/mmr.2015.4376. PMid:26458419.

Kim, S. A., \& Choi, H. C. (2012). Metformin inhibits inflammatory response via AMPK-PTEN pathway in vascular smooth muscle cells. Biochemical and Biophysical Research Communications, 425(4), 866872. http://dx.doi.org/10.1016/j.bbrc.2012.07.165. PMid:22898050.

Livak, K. J., \& Schmittgen, T. D. (2001). Analysis of relative gene expression data using real-time quantitative PCR and the 2(-Delta Delta C(T)). Method. Methods, 25(4), 402-408. http://dx.doi. org/10.1006/meth.2001.1262. PMid:11846609.

Ma, X., Liu, L., \& Meng, J. (2017). MicroRNA-125b promotes neurons cell apoptosis and Tau phosphorylation in Alzheimer's disease. Neuroscience Letters, 661, 57-62. http://dx.doi.org/10.1016/j. neulet.2017.09.043. PMid:28947385.

Marik, P. E. (2014). Early management of severe sepsis: concepts and controversies. Chest, 145(6), 1407-1418. http://dx.doi.org/10.1378/ chest.13-2104. PMid:24889440.

Newton, B., Bhat, B. V., Dhas, B. B., Mondal, N., \& Gopalakrishna, S. M. (2016). Effect of zinc supplementation on early outcome of neonatal sepsis: a randomized controlled trial. Indian Journal of Pediatrics, 83(4), 289-293. http://dx.doi.org/10.1007/s12098-0151939-4. PMid:26616409.

Pedrazza, L., Lunardelli, A., Luft, C., Cruz, C. U., Mesquita, F. C., Bitencourt, S., Nunes, F. B., \& Oliveira, J. R. (2014). Mesenchymal stem cells decrease splenocytes apoptosis in a sepsis experimental model. Inflammation Research, 63(9), 719-728. http://dx.doi. org/10.1007/s00011-014-0745-1. PMid:24888322.

Petry, F. R., Pelletier, J., Bretteville, A., Morin, F., Calon, F., Hébert, S. S., Whittington, R. A., \& Planel, E. (2014). Specificity of anti-tau antibodies when analyzing mice models of Alzheimer's disease: problems and solutions. PLoS One, 9(5), e94251. http://dx.doi. org/10.1371/journal.pone.0094251. PMid:24788298. 
Wang, M., Wu, H., Li, S., Xu, Z., Li, X., Yang, Y., Li, B., Li, Y., Guo, J., \& Chen, H. (2017). SYNJ2BP promotes the degradation of PTEN through the lysosome-pathway and enhances breast tumor metastasis via PI3K/AKT/SNAI1 signaling. Oncotarget, 8(52), 89692-89706. http://dx.doi.org/10.18632/oncotarget.21058. PMid:29163781.

Wei, S., Gao, Y., Dai, X., Fu, W., Cai, S., Fang, H., Zeng, Z., \& Chen, Z. (2019). SIRT1-mediated HMGB1 deacetylation suppresses sepsisassociated acute kidney injury. American Journal of Physiology. Renal Physiology, 316(1), F20-F31. http://dx.doi.org/10.1152/ ajprenal.00119.2018. PMid:30379096.
Zhang, W. X., He, B. M., Wu, Y., Qiao, J. F., \& Peng, Z. Y. (2018). Melatonin protects against sepsis-induced cardiac dysfunction by regulating apoptosis and autophagy via activation of SIRT1 in mice. Life Sciences, 217, 8-15. http://dx.doi.org/10.1016/j.lfs.2018.11.055. PMid:30500551.

Zhu, X., Shao, Z. H., Li, C., Li, J., Zhong, Q., Learoyd, J., Meliton, A., Meliton, L., Leff, A. R., \& Vanden Hoek, T. L. (2014). TAT-protein blockade during ischemia/reperfusion reveals critical role for p85 PI3KPTEN interaction in cardiomyocyte injury. PLoS One, 9(4), e95622. http://dx.doi.org/10.1371/journal.pone.0095622. PMid:24752319. 